\title{
Determining the Efficiency of Malaria Rapid Diagnostic Test to Diagnose Post Transfusion Malaria in Children Under Five Years Old at Komfo Anokye Teaching Hospital
}

\author{
Djeneba Bouare ${ }^{1,}$, Owusu Ofori Alex², Mohammed Abdul-Rahim², Der Muonir Edmund ${ }^{3}$ \\ ${ }^{1}$ School of Medicine and Health Sciences, University for Developement Studies, Tamale, Ghana \\ ${ }^{2}$ Kumasi Centre for Collaborative Research (KCCR), Kumasi, Ghana \\ ${ }^{3}$ Department of Pathology, School of Medicine and Health Sciences, University for Development Studies, Tamale, Ghana
}

Email address:

Bouare.djez@gmail.com (D. Bouare)

*Corresponding author

\section{To cite this article:}

Djeneba Bouare, Owusu Ofori Alex, Mohammed Abdul-Rahim, Der Muonir Edmund. Determining the Efficiency of Malaria Rapid Diagnostic Test to Diagnose Post Transfusion Malaria in Children Under Five Years Old at Komfo Anokye Teaching Hospital. Central African Journal of Public Health. Vol. 6, No. 3, 2020, pp. 122-130. doi: 10.11648/j.cajph.20200603.13

Received: February 19, 2020; Accepted: March 3, 2020; Published: March 17, 2020

\begin{abstract}
Background: Post-transfusion malaria in children under five years is associated with increased morbidity and mortality. The RDTs currently are used to supplement microscopy in the diagnosis of malaria in many endemic countries. However their efficiency as a screening tool for the detection of malaria in donors or in transfusion recipients has not been well evaluated. This study aimed to determine the efficiency of malaria RDT in the detection of post transfusion malaria. Material and methods: The study was conducted from November 2014 to April 2015. Blood samples from 179 patients and their corresponding donors were tested for Plasmodium parasitaemia using RDT and microscopy. The recipients' blood samples were obtained within 48 hours after transfusion for malaria diagnosis. To determine the incidence of post transfusion malaria, 32 patients were purposefully selected. Their pre-transfusion, post transfusion and donor blood samples were tested for malaria using PCR. Results: Approximately 52\% of the study population were within the age group of $0-<1$ year. Prevalence of malaria in blood donors was $27 \%$ by RDT and $7 \%$ by microscopy. Prevalence at pre-transfusion was $34 \%$ by RDT and $13 \%$ by microscopy. Prevalence at post transfusion was $59 \%$ by RDT and $4 \%$ by microscopy. Incidence of post transfusion malaria by PCR was $57 \%$. The sensitivity of RDT in detecting malaria at post transfusion was $100 \%$. Conclusion: Efficiency of malaria RDT as a tool for malaria diagnosis at post transfusion, among children under five years old is good. Clinicians should consider implementing the use of malaria RDT at post transfusion.
\end{abstract}

Keywords: Under-Five, Blood Transfusion-Associated Malaria, Post-Transfusion, RDT, PCR, Kumasi

\section{Introduction}

Malaria is one of the major causes of child death in Africa [1]. According to the latest estimates of World Health Organization (WHO), 198 million cases of malaria occurred globally and the disease led to 584000 deaths. The burden is heaviest in Africa, where an estimated $90 \%$ of all malaria deaths occur, and in children aged under 5 years, who account for $78 \%$ of all deaths [2].

In Ghana, malaria is a burden among children under five years old [3]. An estimated 3.1 to 3.5 million cases of clinical malaria are reported annually in public health facilities, of which 20,000 cases were children under five years old [3, 4]. Malaria is one of the leading causes of childhood death in Ghana [3] with blood transfusion being one of the major sources of malaria transmission $[5,6]$.

Malaria occurring after transfusion could simply be described as post transfusion malaria, and this could be due to parasites acquired by mosquitos' bites or could be acquired from the transfused blood. When post transfusion malaria is due to parasites that were in the donor blood, it is described as transfusion transmitted malaria (TTM). Evidence exists of TTM that has occurred both in Ghana and worldwide [5, 6]. In Sub 
Saharan Africa the demand for blood transfusion is high especially among children under five years and pregnant women $[7,8]$. Blood donors are not screened for malaria in Sub Saharan Africa and Ghana, despite being recommended by WHO [9]. In order to prevent post transfusion malaria, anti-malarial treatment is sometimes given to recipients after transfusion, in Sub Saharan Africa $[10,11]$. Post transfusion malaria can have an impact on the health of non-immune children with complications ranging from severe anaemia to death [11]. Several reasons have been advanced for why, it is difficult to routinely screen blood donors for malaria, in Ghana and the rest of Sub Saharan Africa. Some of the reasons are the non-availability of a sensitive malaria screening method by blood bank services and the severe blood shortages which are already widespread and may be exacerbated by rejecting blood that contains malaria parasites, as asymptomatic carriers are common in endemic areas [12].

The available routine malaria screening methods in malaria endemic areas are light microscopy and malaria RDTs. Malaria RDTs are immune chromatographic tests made to detect the antigens of malaria parasites [13]. They could be specific to one species using a monoclonal antibody or specific to more than one species using a polyclonal antibody [14]. The use of malaria RDTs is widespread nowadays for it is not time consuming, does not require skilled labour and it is cost effective $[15,16]$. Malaria RDTs could however give false positive and false negative results [17].

Microscopy is the gold standard for malaria diagnosis [18]. It helps to detect the presence of malaria parasites from peripheral blood smears. Hence it requires a skilled person and a very well stained slide. However it is time consuming, tedious and cannot be used at most peripheral areas where there is a lack of electricity [19]. The sensitivity of microscopy decreases when the parasitaemia is low [20].

Up to now, there is no evidence based guidance to indicate which malaria screening methods are effective for use by transfusion services [21]. More consideration must then be given to post transfusion malaria especially among children under five years old in order to increase the continuous management of childhood illnesses and death [3, 22].

Microscopy and malaria RDT are the malaria diagnosis tools available in KATH. Microscopy being the gold standard for malaria diagnosis has been the method that is frequently used in KATH. In KATH there is no data on post transfusion malaria diagnosis. If the efficiency of malaria RDT to diagnose post transfusion malaria is shown to be acceptable, physicians will now be able to confirm malaria in transfused recipients before treatment. This could lead to the prompt treatment of recipients, improvement in compliance and the reduction of malaria drugs resistances.

\section{Materials and Methods}

\subsection{Blood Samples Testing Methods}

Blood sample collection from recipients registered into the study.

The recipients were subjected to blood transfusion after a laboratory diagnosis confirming the need for blood transfusion. After enrolment of a child expected to receive blood for transfusion, $1 \mathrm{ml}$ of blood was drawn before the child received the transfusion. Blood collection was done with a $2 \mathrm{ml}$ sterile syringe or a sterile lancet, either through venous, heel or third finger prick. The blood was transferred into an ethylene diamine tetra-acetate (EDTA) tube. The tube was then labelled and sent to the laboratory for malaria testing using malaria RDT, microscopy and PCR. Within 48 hours after blood transfusion the same procedure was performed on the same recipient. Post transfused blood was collected, transferred into an EDTA tube, labelled and sent to the laboratory for malaria diagnosis.

Blood sample collection from blood donors

About $1 \mathrm{ml}$ of donor's blood, which was going to be transfused to the corresponding recipient, was collected from the laboratory section of the TMU. The blood was transferred into an EDTA tube labelled with the same number of the corresponding recipient (e.g.: $\mathrm{B} 1=$ blood given to $\mathrm{P} 1$ ) and sent to the laboratory for malaria testing.

\subsection{Blood Samples Testing Methods}

Blood samples processing for malaria diagnosis using malaria $R D T$

In the laboratory, blood samples (pre-transfusion and posttransfusion blood samples) were tested using a malaria RDT test kit following the manufacture instructions. The test kit was made to detect $P$. falciparum and pan species ( $P$. ovalae, malaria and vivax). When in addition to the control line, a pink/ red line appeared at the $P$. falciparum region the test was reported as $P$. falciparum positive. When the line appeared at the pan region the test was reported as pan positive, and it was reported as $P$. falciparum \& pan positive when 2 distinct lines appeared at the $P$. falciparum and pan's regions. A negative result was recorded when there was no additional line to the control line. No invalid result was recorded. An invalid result would have been reported if there was no line seen. These procedures were performed following the manufacturer instructions (Figure 1).

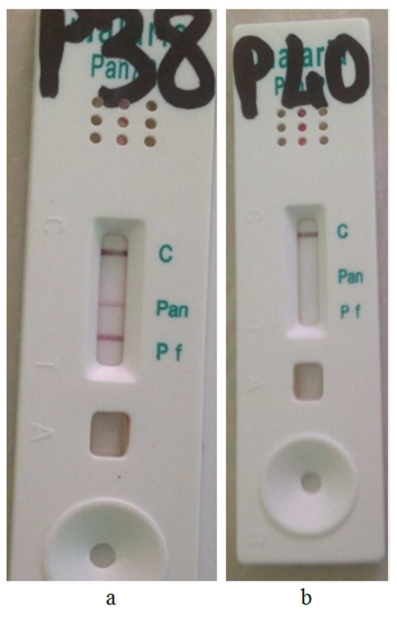

Figure 1. Sample of malaria RDT result; $a=P$. falciparum and pan positive, two distinct lines appeared at the $P$. falciparum $(P . f)$ region and pan region in addition to the control line; $b=n e g a t i v e ~ r e s u l t$, only the control line showed no line at the $P$. falciparum region neither the pan region hence malaria negative result. 
Blood samples processing for malaria diagnosis using microscopy

Thick and thin blood smears were prepared in the laboratory from the same blood used for the malaria RDT. A pipette was used to transfer two distinct drops of blood sample from a labelled EDTA tube unto a clean glass slide. Thick and thin films were prepared on the same slide. The slide was labelled appropriately with the code attributed to the EDTA tube.

Thick film was used to detect the presence or absence of malaria parasites and thin film was used to identify the malaria species.

The thin film was air dried and fixed by dipping into $100 \%$ methanol for about 10 seconds and then air dried again while the thick film was air dried without fixing into methanol. The smears were then stained with a freshly prepared 5\% Giemsa stain solution for 45 minutes and washed with clean distilled water. The slide was then placed on a drying rack and allowed to air dry.

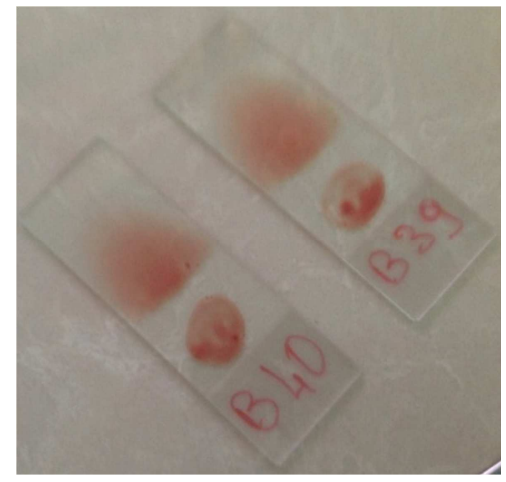

Figure 2. Microscopy slides (thick and thin smears).

\subsection{Blood Samples Processing for Plasmodium Falciparum DNA Detection Using PCR}

\section{Extraction of DNA from blood sample}

The extraction of DNA was performed on 96 samples using the Genotype DNA isolation kit ( $n^{\circ} 51548$, Hain Life science, Germany). The DNA was extracted from $50 \mu \mathrm{l}$ of whole blood by following the manufacturer's instructions. The purified DNA was then stored at $-20^{\circ} \mathrm{C}$.

Plasmodium falciparum DNA identification

The 96 extracted DNA samples were tested by PCR, targeting the Plasmodium small subunit ribosomal RNA (ssrRNA) gene, with 664-693 indicating the position of the forward primer (FAL 1) and 840-869 indicating the position of the reverse primer (FAL 2) for the detection of $P$. falciparum DNA. The PCR was composed of two amplification cycles.

The first amplification was called primary PCR. It was done in order to increase the quantity of template for the nested PCR which was the second amplification cycle. The primary PCR amplified the DNA targeting all the human plasmodium species including $P$. falciparum.

The second PCR called nested PCR was specific only to $P$. falciparum. The product of the primary PCR was then used to amplify only the gene specific to $P$. falciparum.

For the first amplification cycle called primary PCR, $42.5 \mu 1$ of the master mix 1 was prepared for the testing of 1 sample. The reagents were brought out from the freezer ($10^{\circ} \mathrm{C}$ ) thawed at room temperature and shortly centrifuged before they were used. The master mix 1 was composed of following reagents in Table 1 for testing of 1 sample.

Table 1. Composition of the master mix 1 for the primary PCR.

\begin{tabular}{ll}
\hline Reagents & Volume $(\boldsymbol{\mu l})$ \\
\hline Buffer & 10 \\
Magnesium & 8 \\
dNTPs & 4 \\
rPLU1 (forward) & 5 \\
5'TCAAAGATTAAGCCATGCAAGTGA3' & \\
rPLU5 (reverse) 5'CCTGTTGTTGCCTTAAACTCC3' & 5 \\
Tap polymerase & 0.25 \\
Nuclease free water & 10.25 \\
Total & $42.5 \mu 1$ \\
\hline
\end{tabular}

KEY: rPLU1 and rPLU5 indicate the primers used in the primary PCR for the amplification of the DNA to all the plasmodium species. dNTPs indicates dinucleotide triphosphates.

The solution of $42.5 \mu \mathrm{l}$ of the master mix 1 was shortly centrifuged and transferred into a labelled PCR's tube. Afterward, $7.5 \mu \mathrm{l}$ of the extracted DNA (sample) was added to the $42.5 \mu \mathrm{l}$ of the master mix 1 . The DNA amplification was then performed into a thermal cycler with the following steps; initial denaturation $\left(94^{\circ} \mathrm{C}, 4 \mathrm{~min}\right)$, a cycling of 5 : denaturation $\left(94^{\circ} \mathrm{C}, 30 \mathrm{~s}\right)$, hybridization $\left(60^{\circ} \mathrm{C}, 1 \mathrm{~min}\right)$, elongation $\left(72^{\circ} \mathrm{C}, 1 \mathrm{~min}\right)$, a cycling of 30 : denaturation $\left(94^{\circ} \mathrm{C}\right.$, $30 \mathrm{~s})$, hybridization $\left(55^{\circ} \mathrm{C}, 1 \mathrm{~min}\right)$, elongation $\left(72^{\circ} \mathrm{C}, 1 \mathrm{~min}\right)$, followed by a final elongation $\left(72^{\circ} \mathrm{C}, 4 \mathrm{~min}\right)$ and a conservation stage $\left(10^{\circ} \mathrm{C}\right.$, unlimited time). For the nested PCR, a master mix 2 was prepared. It was composed of the following reagents in Table 2 for the testing of 1 sample.

Table 2. Composition of the master mix 2 for the nested PCR.

\begin{tabular}{lll}
\hline Reagents & Volume $\boldsymbol{\mu l}$ \\
\hline Buffer Blue & 4 \\
Magnesium & 3.2 \\
dNTPs & 1.6 \\
FAL 1 (forward) 5'TTAAACTGGTTTGGGAAAACCAAATATATT3' & 2 \\
FAL 2 (reverse) 5'ACACAATGAACTCAATCATGACTACCCGTC3' & 2 \\
Taq polymerase & 0.1 \\
Nuclease free water & 5.1 \\
Total & $18 \mu 1$ \\
\hline
\end{tabular}

KEY: FAL 1 and FAL 2 indicate the primers used for the amplification of only $P$. falciparum gene. dNTPs indicates dinucleotide triphosphates. 
The solution of $18 \mu$ l of the master mix 2 was shortly centrifuged and then transferred into a labelled PCR's tube. Afterward the $2 \mu \mathrm{l}$ of the product of the primary PCR which was the master mix 1 was added to the labelled PCR's tube containing the master mix 2. The DNA amplification was then performed into a thermal cycler with the following steps; initial denaturation $\left(94^{\circ} \mathrm{C}, 4 \mathrm{~min}\right)$, a cycling of 5 : denaturation $\left(94^{\circ} \mathrm{C}, 30 \mathrm{~s}\right)$, hybridization $\left(63^{\circ} \mathrm{C}, 1 \mathrm{~min}\right)$, elongation $\left(72^{\circ} \mathrm{C}, 1 \mathrm{~min}\right)$, a cycling of 30 : denaturation $\left(94^{\circ} \mathrm{C}\right.$, $30 \mathrm{~s})$, hybridization $\left(58^{\circ} \mathrm{C}, 1 \mathrm{~min}\right)$, elongation $\left(72^{\circ} \mathrm{C}, 1 \mathrm{~min}\right)$ followed by a final elongation $\left(72^{\circ} \mathrm{C}, 4 \mathrm{~min}\right)$ and a conservative stage $\left(10^{\circ} \mathrm{C}\right.$, unlimited time $)$. The final product of the PCR was then sent to the Gel room for electrophoresis and bands' detection.

\section{Electrophoresis and band detection}

The final product of the PCR was detected through electrophoresis on $1.5 \%$ agarose gel. The casting tray was prepared and the comb fixed in position. An amount of $1.2 \mathrm{~g}$ of agarose gel were weighted and poured in a volume of $80 \mathrm{ml}$ of 1xTris-borate with EDTA (TBE composed of Tris base, Boric acid and EDTA). The solution was mixed well and heated for 2 minutes, after its temperature was cooled down under running water. When the gel had cooled to the point that it could be comfortably touched, $3 \mu \mathrm{l}$ of ethidium bromide was added and uniformly mixed with the agarose. The gel was carefully poured into the casting tray. All air bubbles were removed and the gel allowed to solidify. After solidification the comb was removed and the gel was placed in the electrophoresis tank. A volume of $8 \mu \mathrm{l}$ of the final product of the PCR was loaded into the wells. Afterward, $5 \mu 1$ of $100 \mathrm{bp}$ ladder (Invitrogen) was loaded into the first well (starting from the left). The positive and negative controls were the last to be loaded. Then an electric current of $100 \mathrm{~V}$ were applied to the gel for 30 minutes. Image of the gel was captured, using a Trans-illuminator, with the software infinity Vx2. The bands were detected under UV light (Figure 3).

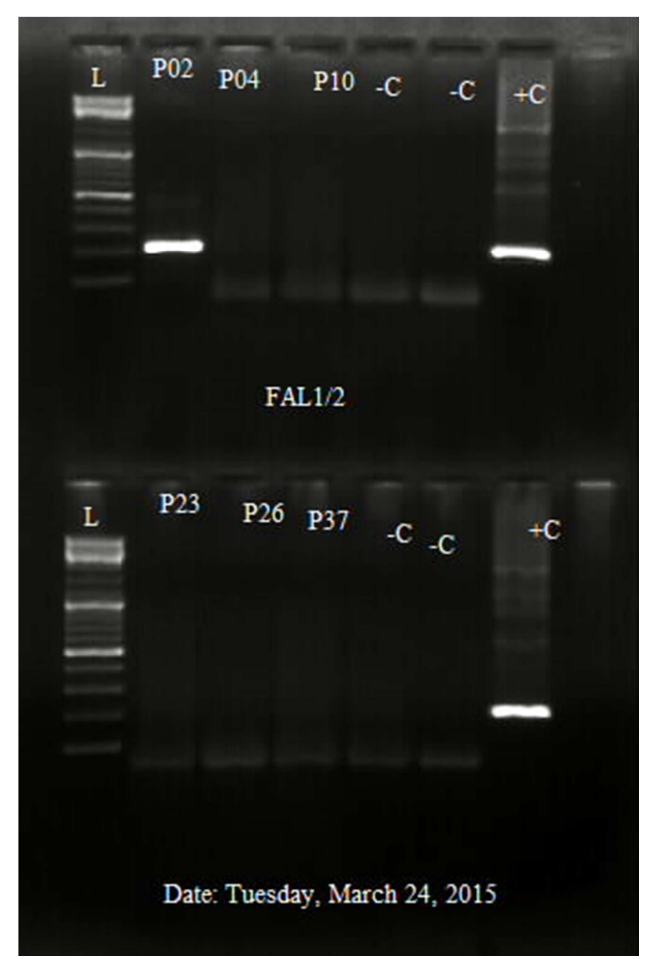

Figure 3. $1.5 \%$ agarose gel analysis of nested PCR product.

( $\mathrm{L}=$ ladder; P02, P04, P23, P26 and P37=pre transfusion samples of patients number $02,04,23,26$ and 37 respectively; $-\mathrm{C}=$ negative control; $+\mathrm{C}=$ positive control; $\mathrm{FAL}=$ falciparum).

\section{Results}

\section{Recipients' characteristics}

A total of 179 children under five years old were recruited into the study. Their ages ranged from $0-4$ years, with a mean age of 1.37 years and a modal age group of $0-<1$ $(52 \%)$ year.

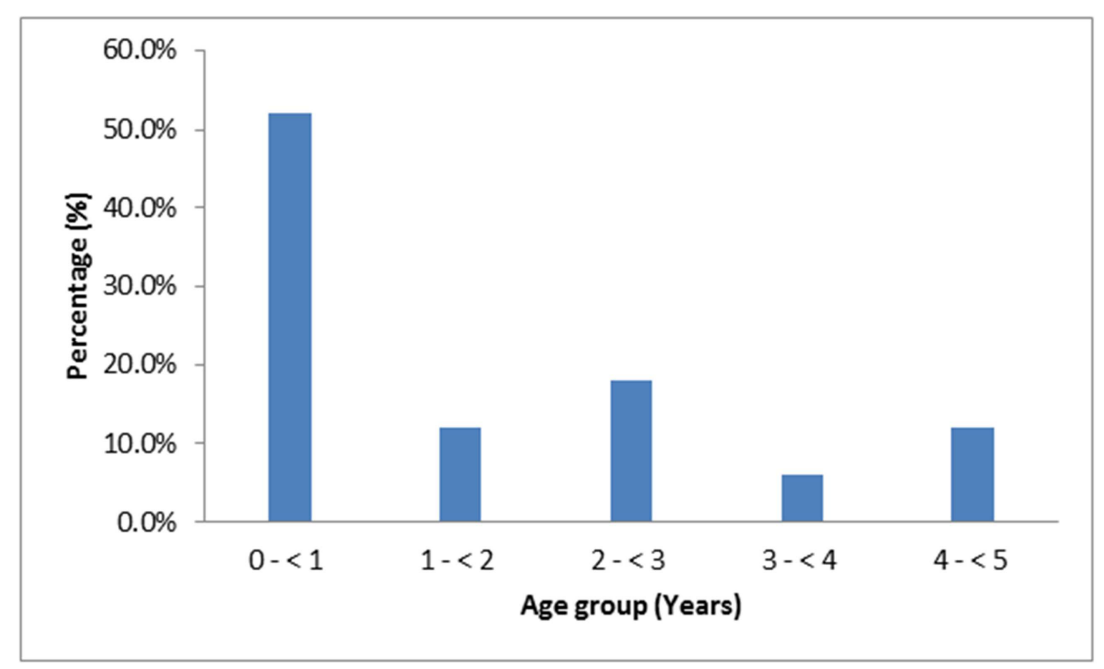

Figure 4. Age characteristic of the study population.

The majority (44\%) were from mother and baby unit (MBU) (Table 1). Many (64.0\%) of the children were males
(Table 1).

At the time of enrolment, 76 recipients $(42 \%)$ had been 
tested for malaria in the wards using malaria RDT $\left(1^{\text {st }}\right.$ response malaria RDT) which detected only $P$. falciparum infection (Table 3). Among those that tested negative, $17 \%$ were given anti-malaria drugs, contrary to WHO treatment guidelines. There were 103 (58\%) who were not tested for malaria in the wards. Contrary to WHO guidelines, $17 \%$ were also given anti-malaria drugs. The most frequently used anti-malarial drugs was Artemisinin-based Combination Therapy (ACT) (74\%) followed by camoquine (18\%), artemether Lumefantrine (6\%) and quinine (2\%).

Table 3. Characteristics of the 179 recipients recruited into the study.

\begin{tabular}{lll}
\hline Characteristics & & N (\%) \\
\hline \multirow{3}{*}{ Wards } & MBU & $79(44 \%)$ \\
& PEU & $62(35 \%)$ \\
Sex & Others & $38(21 \%)$ \\
& Male & $115(64 \%)$ \\
& Female & $64(36 \%)$ \\
Recipients tested & Malaria positives & $76(42 \%)$ \\
for malaria & Treatment with anti-malarial & $41(54 \%)$ \\
& Malaria negative & $41(54 \%)$ \\
Recipients not & Treatment with anti-malarial & $35(46 \%)$ \\
tested for malaria & Treatment with anti-malarial & $103(17 \%)$ \\
\hline
\end{tabular}

Study drop out

Post-transfusion samples could not be collected from 58 $(32 \%)$ of those enrolled. Death $(36 \%)$ accounted for the major reason why these samples were not collected (Table 4). Seven $(12 \%)$ who were due to be transfused were no more transfused as required. There were 6 recipients $(10 \%)$ who were transfused twice the same day. Another group of 6 children $(10 \%)$ could not be traced after transfusion. Five children (9\%) were discharged the same day after blood transfusion. A group of 4 children (7\%) had their clinical condition deteriorating, therefore taking further blood sample was deemed unethical. Only $1(2 \%)$ was wrongly enrolled into the study, the correct age of 6 years was discovered after the enrolment (Table 4).

Table 4. Reasons for why recipients did not have a post transfusion sample collected.

\begin{tabular}{ll}
\hline Reasons of drop out & N (\%) \\
\hline Death & $21(36 \%)$ \\
Blood transfusion stopped & $7(12 \%)$ \\
Consent withdrawals & $6(10 \%)$ \\
Transfused twice the same day & $6(10 \%)$ \\
Could not traced after blood transfusion & $6(10 \%)$ \\
Discharged before second sample collection & $5(9 \%)$ \\
Clinical condition worsened & $4(7 \%)$ \\
Wrongly enrolled & $1(2 \%)$ \\
\hline
\end{tabular}

\section{Prevalence of malaria}

Among the 179 pre-transfusion samples collected from recipients, prevalence of malaria by malaria RDT was $34 \%$ (60/179). Out of those 60 positive samples, 19 (32\%) were mixed infection for P. falciparum and P. ovale or P. malarae or P. vivax. The other 41 (68\%) samples were only $\mathrm{P}$. falciparum positive. The prevalence of malaria by microscopy was $13 \%$ (24/179) (Table 3). P. falciparum was the only species identified after speciation when light microscopy was used.

The prevalence of malaria among the 179 samples collected from blood donors, using malaria RDT was $27 \%$ (49/179) (Table 3). Among those positive, 46 (94\%) were P. falciparum positive, $2(4 \%)$ were mixed infection with $\mathrm{P}$. falciparum and P. ovale or P. malarae or P. vivax, 1 (2\%) was mixed infection with P. ovale or P. malarae or P. vivax. The prevalence of malaria by microscopy was $7 \%(12 / 179)$ and $P$. falciparum was the only species identified.

There were 121 post-transfused samples tested for malaria. The prevalence of malaria at post transfusion, by malaria RDT was $59 \%$ (71/121). Out of the 71 positives, 2 (3\%) were mixed infection with $P$. falciparum and $P$. ovale or $P$. malarae or $P$. vivax. The other $69(97 \%)$ were only $P$. falciparum positive. The prevalence of malaria by microscopy was $4 \%$ $(5 / 121)$ (Table 5).

Table 5. Comparison of the prevalence of malaria by malaria RDT and microscopy.

\begin{tabular}{|c|c|c|c|c|c|}
\hline & Test methods & Number of samples tested & Number of positive & Prevalence & P-values fpr positive cases \\
\hline \multirow{2}{*}{ Pre transfusion } & RDT & 179 & 60 & $34 \%$ & \\
\hline & Microscopy & 179 & 24 & $13 \%$ & \\
\hline Donors & RDT & 179 & 49 & $27 \%$ & \\
\hline Post transfusion (within & RDT & 121 & 71 & $59 \%$ & \\
\hline $48 \mathrm{H}$ after transfusion) & Microscopy & 121 & 5 & $4 \%$ & \\
\hline
\end{tabular}

\section{Incidence of post-transfusion malaria.}

The incidence of post-transfusion malaria was determined from the 32 selected recipients diagnosed for $P$. falciparum infection using PCR. Among the 32 recipients, 28 were negative at pre transfusion, 21 of their corresponding donors' blood were $P$. falciparum positive and 16 became positive at post transfusion. At post-transfusion, the condition of those 16 recipients was recorded from their folders. Only $1(6 \%)$ was reported to be in critical condition, $10(63 \%)$ were febrile and pale and no symptoms was recorded on the other $5(31 \%)$ recipients. The incidence of post-transfusion malaria by PCR was $57 \%(16 / 28)$. Among the other 5 recipients, though they received positive $P$. falciparum blood, were negative at post transfusion. Three were treated with ACT at post transfusion. The other 2 were also negative at post transfusion though they did not receive any anti-malarial treatment.

They were 7 recipients out of the 28 were negative at pretransfusion, received negative $P$. falciparum blood and remained negative at post-transfusion.

Determination of the efficiency of malaria RDT to microscopy in detecting malaria at post transfusion 
With PCR as the gold standard, the sensitivity of malaria RDT in detecting $P$. falciparum at post transfusion was $100 \%$ compared to $5.2 \%$ by microscopy. However microscopy had the highest specificity of $100 \%$ while malaria RDT had
$15.3 \%$. The negative predictive value was $100 \%$ by RDT while microscopy had a $100 \%$ of positive predictive value (Table 6).

Table 6. Comparison of the efficiency of malaria RDT to microscopy in detecting post transfusion malaria.

\begin{tabular}{|c|c|c|c|c|c|c|}
\hline Post transfusion & PCR & & Sensitivity & Specificity & Positive predictive value & Negative predictive value \\
\hline \multirow{2}{*}{$\begin{array}{l}\text { (within } 48 \mathrm{H} \text { after } \\
\text { transfusion) }\end{array}$} & \multirow{2}{*}{+ ve } & \multirow{2}{*}{$-v e$} & $\%$ & $\%$ & $\%$ & $\%$ \\
\hline & & & $(95 \% \mathrm{CI})$ & $(95 \% \mathrm{CI})$ & $(95 \% \mathrm{CI})$ & $(95 \%)$ \\
\hline \multicolumn{7}{|l|}{ RDT } \\
\hline -ve & 0 & 2 & $(79-100)$ & $(2.7-46.3)$ & $(43.9-79.4)$ & $(19.7-100)$ \\
\hline \multicolumn{7}{|l|}{ Microscopy } \\
\hline$+\mathrm{ve}$ & 1 & 0 & 5.2 & 100 & 100 & 41.9 \\
\hline
\end{tabular}

\section{Discussion}

\section{Incidence of post transfusion malaria}

The incidence of post transfusion malaria by PCR was $57 \%$ in single transfused recipients. This was high, compared to the incidences of $2.9 \%$ of post transfusion malaria reported in single transfused recipients and $4.9 \%$ in multiple transfused recipients in Sudan, by Ali et al., (2004) [23] using microscopy as the only test method. The differences could be due to the sensitivity of the test methods used because; PCR is more sensitive than microscopy. The results of this study show that, post transfusion malaria is a real health problem in Ghana and hence blood for transfusion must be carefully investigated to prevent malaria associated transfusion complications such as severe anaemia or death [11]. The health impact is even worse among non-immune children leading to complications such as severe anaemia or death [11].

Among those 21 recipients who received $P$. falciparum infected blood, 5 recipients were negative at post transfusion. Three of them received ACT as anti-malarial at post transfusion. This anti-malarial drug is known to be the fastest acting anti-malarial available. It inhibits the development of the trophozoites and thus prevents progression of the disease. Young circulating parasites are killed before they sequester in the deep microvasculature. This drug starts acting within 12 hours. These properties of the drug are very useful in managing P. falciparum malaria [17]. This could explain why the 3 recipients were detected negative within 48 hours at post transfusion though they received positive donors' blood.

However, anti-malarial drugs did not influence the results of malaria RDT at post-transfusion. This means that, recipients who were negative at pre-transfusion were detected malaria positive at post transfusion by malaria RDT after receiving a positive malaria donors' blood.

The post-transfusion malaria cases in this study were likely to be of TTM because all the 16 positive $P$. falciparum post transfused recipients were initially negative at pre transfusion and became positive after being transfused with a positive $P$. falciparum blood. This shows that children under five years old in KATH could be at risk of TTM which can led to severe complications of malaria because of their low level of immunity against the disease, malaria.

Among the 32 samples subjected to PCR, 7 were negative at pre transfusion, received negative $P$. falciparum blood and remained negative at post transfusion. Based on these results showed, no likely environmental post transfusion malaria case was detected in this study.

Sensitivity of malaria RDT and microscopy

In post-transfused samples, malaria RDT had a sensitivity of $100 \%$ while microscopy had $5.2 \%$. In this study, the sensitivity of $100 \%$ showed by malaria RDT conformed to WHO recommendations for malaria RDT performance (>90\%) [16]. Endeshaw et al, (2008) [24] found that HRP-2 based $P$. falciparum-specific tests, generally have good sensitivity (over 90\%). A similar study on the efficiency of RDT, by Gatti et al., (2007), [25] showed malaria RDT to be more sensitive (100\%) than microscopy $(91 \%)$. The current finding further supports the sensitivity of $98 \%$ of malaria RDT reported in India by Bahadur et al (2010) study [26].

Over all malaria RDTs are sensitive for malaria diagnosis comparing to microscopy $[27,28]$ either in malaria diagnosis or malaria cases management [29] in malaria endemic areas such as Ghana [30]. Malaria RDTs offer higher sensitivity ( $>90 \%$ for P. falciparum) than microscopy [14].

Although microscopy remains the gold standard for malaria diagnostic, $[18,31]$ RDTs offer a good alternative [32].

\section{Feasibility of the implementation of malaria RDT}

In this study, malaria RDT had shown high sensitivity. If implemented as a screening tool it will efficiently be able to detect parasitaemia. What becomes of malaria positive blood donors or? We cannot afford to waste blood, because of the already severe blood shortages in malaria endemic areas ${ }^{12}$ and the transfusion of a known infected blood could result in ethical issues. Consideration also have to be made whether the positive malaria blood donors will be treated or not or if they will have to buy their own medicine. Malaria RDT detects the antigen of $P$. falciparum even in the absence of viable parasites. These are some of the challenges in implementing malaria RDT for use at blood bank services.

According to Masanja et al, (2012) [31] and Boyce et al (2015) [33], the implementation of malaria RDT at posttransfusion will increase the use of RDT for malaria confirmation and reduce the prophylaxis treatment with ACT 
given to recipients at post transfusion without any laboratory diagnosis, which goes against WHO suggestion [34]. Besides Gerstl et al, (2010) [35] supported the wider use of malaria RDT to accurately diagnose malaria and avoid over and under treatment of malaria. They also refuted the idea that, malaria RDT may miss a significant number of true positive among children under five years old. An effective treatment of malaria, depends on a laboratory diagnosis [27].

The implementation of malaria RDT testing at post transfusion will improve differential diagnosis and fever management, ensure prompt treatment of recipients, diminish unnecessary use of ACTs and restrict the spread of malaria drugs resistances [36]. Also it will reduce the administration of malaria drugs without diagnosis in Ghana, reducing recipients' expenditures [37] and the risk of children exposed to unnecessary treatment [38].

Presumptive anti-malarial treatment at post transfusion

The practice in KATH shows that some $17 \%$ of malaria negative transfused recipients and similarly $17 \%$ of those not investigated for malaria were given anti-malarial treatment.

This practice in KATH was also confirmed by Owusu-Ofori (2012) [5] where anti-malarial treatment were given despite a negative laboratory test. This goes against WHO recommendations which suggest treatment after laboratory confirmation [33]. These results showed that the recommendations are not always met by the physicians. However studies are needed to understand why this practice continues.

Comparison of humans Plasmodium antigens

The HRP2 is water-soluble protein expressed only by $P$. falciparum trophozoites. It persists in the circulating blood after the parasitaemia has cleared or has been greatly reduced. It generally takes around two weeks or months after successful treatment for HRP2-based tests to turn negative [39]. Since HRP-2 is expressed only by P. falciparum, these tests will give negative results with samples containing only $P$. vivax, $P$. ovale, or $P$. malariae. Studies need to be done on how a positive HRP2 result must be interpreted.

Plasmodium lactate dehydrogenase $(\mathrm{pLDH})$ is an enzyme. PLDH does not persist in the blood but clears about the same time as the parasites following successful treatment [40]. The lack of antigen persistence after treatment makes the pLDH test useful in predicting treatment failure. It can be used to detect infection caused by $P$. vivax, $P$. malariae, and $P$. ovale and $P$. falciparum. PLDH detection seems to be the best in the diagnosis of malaria. Plasmodium aldolase is an enzyme produced by all four species (Joel et al., 2013) [15]. The presence of antibodies against the plasmodium aldolase in the sera of human adults partially immune to malaria suggest that plasmodium aldolase is implicated in protective immune response against the parasite.

\section{Conclusion}

Malaria rapid diagnostic test (RDT) is a good tool for the detection of post transfusion malaria. Malaria RDT had been shown to be more effective than microscopy in Plasmodium falciparum malaria detection. Furthermore, it does not need a skilled person to perform the test and the result is obtained within minutes.

\section{Conflict of Interest}

There is no conflict of interest.

\section{Consent to Publish This Case Report}

This was obtained for the ethical committee of the Kumasi Centre for Collaborative Research.

\section{Funding}

The authors received no funding for the work.

\section{Acknowledgements}

We want to acknowledge all staff of the Komfo Anokye Teaching Hospital and the Kumasi Centre for Collaborative Research who helped in one way or the other during the laboratory work and the write-up of this work.

\section{References}

[1] Elphinstone, R. E., Higgins, S. J., \& Kain, K. C. Prevention of Malaria in Travelers: Bite Avoidance and Chemoprophylactic Measures. Current Treatment Options in Infectious Diseases, 2014; 6: 47-57.

[2] World Health Organization (WHO). World malaria report 2014. From www.who.int/malaria/...malaria_report_2014/wmr-2014-noprofiles.pdf. Accessed on July 2015.

[3] Nyarko, S. H., Cobblah, A. Sociodemographic Determinants of Malaria among Under-Five Children in Ghana. Malaria research and treatment, 2014.

[4] Buabeng, K. O., Duwiejua, M., Dodoo, A. N., Matowe, L. K., Enlund, H. Self-reported use of anti-malarial drugs and health facility management of malaria in Ghana. Malaria journal, 2007; 6: 85 .

[5] Owusu-Ofori, A. Transfusion-transmitted malaria and bacterial infections in a malaria endemic region. (2012; Doctoral dissertation, University of Liverpool.

[6] O’Brien et al, 2015 O’Brien, S. F., Delage, G., Seed, C. R., Pillonel, J., Fabra, C. C., Davison, K \& Leiby, D. A. The Epidemiology of Imported Malaria and Transfusion Policy in 5 Nonendemic Countries. Transfusion medicine reviews. 2015.

[7] Austin N, Adikaibe E, Ethelbert O, Chioma U, Ekene N. Prevalence and Severity of Malaria Parasitemie among Children Requiring Emergency, Blood transfusion in tertiary hospital in Imo state, Nigeria. Annals of Medical and Health Science Research. 2014; 4: 19-23.

[8] Erhabor Osaro, Adias Teddy Charles. The challenges of meeting the blood transfusion requirements in Sub-Saharan Africa: the need for the development of alternatives to allogenic blood. Journal of Blood Medicine, 2011; 2: 7-21. 
[9] Mark E. Brecher and Shauna N. Hay. Bacterial Contamination of Blood Components Department of Pathology and Laboratory Medicine, University of North Carolina. Journal of Clinical Microbiololy Review, 2005; 18: 195-204.

[10] Jobert Richie N Nansseu, Jean Jacques N Noubiap, Shalom Tchokfe Ndoula, Albert Frank M Zeh and Chavely Gwladys Monamele. (2013). What is the best strategy for the prevention of transfusion-transmitted malaria in sub-Saharan African countries where malaria in endemic? Malaria Journal, 2013; 12: 1475-2875.

[11] Tayou, T. C., Mbanya, D., Garraud, O., \& Lefrère, J. J. (2007) [Blood safety: malaria and blood donation in Africa]. Transfusion clinique et biologique: journal de la Societe francaise de transfusion sanguine, 2007; 14: 481-486.

[12] Bates, I., Owusu-Ofori, A. K., \& Parry, C. Malaria in the blood supply in Africa: Is it a problem? Vox Sanguinis, 2011; 101: 55-55.

[13] Kátia Luz Torres, Mônica Nascimento dos Santos Moresco, Luciane Rodrigues Sales, Josilene da Silva Abranches, Márcia Almeida Araújo Alexandre and Adriana Malheiro. Transfusion-transmitted malaria in endemic zone: epidemiological profile of blood donors at the Fundação HEMOAM and use of rapid diagnostic tests for malaria screening in Manaus. Rev. Bras. Hematol. Hemoter. 2014; 36 : 269-274.

[14] Weekley, C., \& Smith, D. S. Malaria: the clinical basics. Global Health Education Consortium. 2013.

[15] Joel C. Mouatcho and J. P. Dean Goldring. Malaria rapid diagnostic tests: challenges and prospects. Journal of Medical Microbiology, 2013; 62: 1491-1505.

[16] Chou, M., Kim, S., Khim, N., Chy, S., Sum, S., Dourng, D \& Ménard, D. (2012). Performance of "VIKIA Malaria Ag Pf/Pan" (IMACCESS $®)$ ), a new malaria rapid diagnostic test for detection of symptomatic malaria infections. Malaria Journal, 2012; 11: 10-1186.

[17] Kakkilaya, B. S. Are We Treating Malaria? World, 1990: 3.

[18] Li, Y., Kumar, N., Gopalakrishnan, A., Ginocchio, C., Manji, R., Bythrow, M, Kong, H. Detection and species identification of malaria parasites by isothermal tHDA amplification directly from human blood without sample preparation. The Journal of Molecular Diagnostics, 2013; 15: 634-641.

[19] Mali, S., Kachur, S. P., Arguin, P. M. Malaria surveillanceUnited States, 2010. MMWR Surveill Summ, 2012; 61: 1.

[20] Johnston, S. P., Pieniazek, N. J., Xayavong, M. V., Slemenda, S. B., Wilkins, P. P., da Silva, A. J. PCR as a confirmatory technique for laboratory diagnosis of malaria. Journal of clinical microbiology, 2006; 44: 1087-1089.

[21] Lund, T. C., Hume, H., Allain, J. P., McCullough, J., Dzik, W. The blood supply in Sub-Saharan Africa: Needs, challenges, and solutions. Transfusion and Apheresis Science, 2013; 49: 416-421.

[22] Bhutta, Z. A., Chopra, M., Axelson, H., Berman, P., Boerma, T., Bryce, J \& Wardlaw, T. (2010). Countdown to 2015 decade report (2000-10): taking stock of maternal, newborn, and child survival. The Lancet, volume 375 (9730), pp20322044.

[23] Ali, M. S. M., Kadaru, A. A. G. M. Y., Mustafa, M. S. (2004).
Screening blood donors for malaria parasite in Sudan. Ethiopian Journal of Health Development, 2004; 18: 70-74.

[24] Endeshaw, T., Gebre, T., Ngondi, J., Graves, P. M., Shargie, E. B., Ejigsemahu, Y., et al. Evaluation of light microscopy and rapid diagnostic test for the detection of malaria under operational field conditions: a household survey in Ethiopia. Malaria journal, 2008; 7: 118.

[25] Gatti, M. Gramegna, Z. Bisoffi, A. Raglio1, M. Gulletta, C. Klersy, A. et al., A comparison of three diagnostic techniques for malaria rapid diagnostic test (NOWH Malaria), PCR and microscopy. Annals of tropical medicine and parasitology, 2007; 101: 195-204.

[26] Bahadur, S., Pujani, M., Jain, M. Use of rapid detection tests to prevent transfusion-transmitted malaria in India. Asian journal of transfusion science, 2010; 4: 140.

[27] Akotet, B. M. K., Nkare, C. A., Mbouoronde, O. C., MawiliMboumba, D. P. (2014). Journal. Malaria Chemoth Cont Elimination, 2014; 3: 125

[28] Chanda, P., Hamainza, B., Moonga, H. B., Chalwe, V., Pagnoni, F. (2011). Community case management of malaria using ACT and RDT in two districts in Zambia: achieving high adherence to test results using community health workers. Malaria Journal, 2011; 10: 158.

[29] Asante, K. P., Abokyi, L., Zandoh, C., Owusu, R., Awini, E., Sulemana, A et al. Community perceptions of malaria and malaria treatment behaviour in a rural district of Ghana: implications for artemisinin combination therapy. Public Health, 2010; 10: 409.

[30] Daniel Eibach, Boubacar Traore, Mourad Bouchrik, Boubacar Coulibaly, Nianégué Coulibaly, Fanta Siby, Guillaume Bonnot, Anne-Lise Bienvenu and Stéphane Picot. Evaluation of the malaria rapid diagnostic test VIKIA malaria $\mathrm{Ag}$ $\mathrm{Pf} / \mathrm{Pan}^{\mathrm{TM}}$ in endemic and non-endemic settings. Malaria Journal, 2013; 12: 188.

[31] Masanja, I. M., Selemani, M., Amuri, B., Kajungu, D., Khatib, R., Kachur, S. P., \& Skarbinski, J. (2012). Increased use of malaria rapid diagnostic tests improves targeting of antimalarial treatment in rural Tanzania: implications for nationwide rollout of malaria rapid diagnostic tests. Malaria Journal, 2012; 11: 221.

[32] Mens, P., Spieker, N., Omar, S., Heijnen, M., Schallig, H. D. F. H., Kager, P. A. Is molecular biology the best alternative for diagnosis of malaria to microscopy? A comparison between microscopy, antigen detection and molecular tests in rural Kenya and urban Tanzania. Tropical Medicine \& International Health, 2007; 12: 238-244.

[33] Newman, R. D. World Malaria Report 2011. 2012.

[34] Gerstl, S., Dunkley, S., Mukhtar, A., De Smet, M., Baker, S., Maikere, J. Assessment of two malaria rapid diagnostic tests in children under five years of age, with follow-up of false-positive pLDH test results, in a hyperendemic falciparum malaria area. Sierra Leone. Malaria Journal, 2010; 9: 28 .

[35] Zhao, J., Lama, M., Korenromp, E., Aylward, P., Shargie, E., Filler, S \& Atun, R. (2012). Adoption of rapid diagnostic tests for the diagnosis of malaria, a preliminary analysis of the global fund program data, 2005 to 2010. PloS one, 2012; 7: e43549. 
[36] Ansah, E. K., Narh-Bana, S., Affran-Bonful, H., Bart-Plange, C., Cundill, B., Gyapong, M., et al. The impact of providing rapid diagnostic malaria tests on fever management in the private retail sector in Ghana: a cluster randomized trial. Malaria journal, 2015; 350: 1019.

[37] Reyburn, H., Mbakilwa, H., Mwangi, R., Mwerinde, O., Olomi, R., Drakeley, C., et al. Rapid diagnostic tests compared with malaria microscopy for guiding outpatient treatment of febrile illness in Tanzania: randomised trial. Biomedical journal, 2007; 334: 403.

[38] Boyce, R. M., Muiru, A., Reyes, R., Ntaro, M., Mulogo, E., Matte, M., \& Siedner, M. J. (2015). Impact of rapid diagnostic tests for the diagnosis and treatment of malaria at a peripheral health facility in Western Uganda: an interrupted time series analysis. Malaria Journal, 2015; 14: 203.

[39] Hendriksen, I. C., White, L. J., Veenemans, J., Mtove, G., Woodrow, C., Amos, B etal. Defining falciparum-malariaattributable severe febrile illness in moderate-to-high transmission settings on the basis of plasma PfHRP2 concentration. Journal of Infectious Diseases, 2013; 207: 351361 .

[40] Lassina, Ouattara KLoudou, Jean \& Cheikna, Zongo \& N, Barro \& Savadogo, Aly \& Bassolé, Imael \& A. S, Ouattara \& Traore, Alfred. (2011). Antioxidant and Antibacterial Activities of Three Species of Lannea from Burkina Faso. Journal of Applied Sciences. 11.10.3923/jas.2011.157.162. 